\title{
ANALYSIS OF POLICY MANAGEMENT MODELS AND SPECIFICATION LANGUAGES
}

\author{
Issam Aib, Nazim Agoulmine, Mauro Sergio Fonseca, Guy Pujolle \\ LIP6 Computing Laboratory, University of Paris 6, France (name.surname@lip6.fr)
}

Abstract: In this work, we will focus on the work undertaken in the specification of policies, the different notations developed, and how policies are deployed within a managed system. We will motivate, analyze, and classify all major existing policy notations through several criteria and try to consider the consistent merging of their strengths into a more appropriate framework. We also propose a set of enhancing features to existing policy specification notions namely domain membership rules, role compatibility rules, and light events. The work ends by an enumeration of the set of features which a policy specification language needs to model in order to facilitate the process of implementing adaptive Quality of Service management and rapid deployment of customized services.

Key words: Policy Specification Languages, Policy Notation, QoS Policy Specification, Policy Refinement, Classification of policy notations

\section{INTRODUCTION}

Policy discipline is gaining more and more research and application areas both in industry and academia leading a continuous shift from the traditional client-server model to a service-driven model of network management. In computer networks, a major concern has been to cater for adaptive policybased Quality of Service provisioning. Multiple approaches have been used for this end ranging from manual configuration of per device vendor specific policies of network elements and spreading up to full blown highly (but not

The original version of this chapter was revised: The copyright line was incorrect. This has been corrected. The Erratum to this chapter is available at DOI: 10.1007/978-0-387-35703-4_21

D. Gaït et al. (eds.), Network Control and Engineering for QoS, Security and Mobility II

(C) IFIP International Federation for Information Processing 2003 
fully) automated policy management graphical environments developed mainly within industry. Yet, still there is much to plough in the field.

For the policy approach to management to become effective, policy needs to be clearly specified in a language easily understandable by human administrators and policy-makers but also enforceable on network elements. The language must be applicable to a uniform representation of network elements as well as their properties, operations, and relationships expressed in a device independent information model. To be runnable, the policy specification must be free of conflicts and must match the device capabilities.

As a means of management, a policy definition must conform to a number of requirements [1]. It should be precise regarding its interpretation, consistent in its deployment across system elements and compatible with their respective capabilities. The specification should be at a level of easiness and intuitiveness that simplifies policy expression using an abstract day-to-day activities language. This more user-friendly language helps in generating policies optimized for specific devices.

For now, it is generally accepted that there has to be at least two main levels of policy definition. A Low level Policy (LLP), also described as device driven, should be specified to match device capabilities in order to become effective. A High level Policy (HLP), on the other hand, depends on the end-goal of the network operator and is relatively independent of the underlying technology of the operator's network. Hence, it is said to be business driven.

\section{INTERNET QUALITY OF SERVICE OVERVIEW}

Internet Quality of service, or simply QoS for writing convenience, describes mechanisms by which is assured sufficient performance and service differentiation such as low delay and low packet loss for certain types of applications or traffic [28]. QoS can be described both qualitatively through relative definitions or quantitatively using absolute metrics. It is assured by reserving resources, primarily bandwidth and, sometimes, buffer space for specific types of traffic. In case where excess traffic is encountered either packet level (policing) or flow level control (admission control) is applied in order to ensure sufficient performance for the treatment of prioritized traffic.

To ensure the correct delivery of end-to-end QoS, more than mere network QoS enforcement needs to be done. End host support for QoS, including server QoS and application level adaptation, play also an equally important role. If network QoS is to be provided through the use of a known 
policy discipline, then we should have to consider the emergence of a new type of applications, mainly policy aware applications that can work in harmony within policy based networks. This is facilitated through the existence of a clear policy specification language which serves as the link or communicating tunnel between policy aware networks and policy aware applications.

\section{IETF/DMTF POLICY FRAMEWORK}

Instead of defining a policy notation using a specification language and its underlying grammar, the IETF policy working group preferred to do it directly using UML like object oriented modeling. This constitutes a good choice from the point of view of low-level policy distribution among the management system components and their work serves also in the definition of an overall policy framework in which policy notation is not but a single part of a whole system. However, there is still a big need for an easy to use notation for policy at the high level entry in the system management tool and all other related operations of compilation, validation, and refinement of HLPs, as well as their exchange among different components.

The IETF defined a set of information models to carry up all various aspects of any managed environment. At the top of the hierarchy is the Core Information Model (CIM), which is composed of a small set of classes and associations that establish a conceptual framework for the schema of the rest of the managed environment. Second, comes the Policy Core Information model (PCIM, and recently extended to PCIMe) which tries to define a set of classes and relationships that provide an extensible means for defining policy control of managed objects [6]. PCIM enables administrators to represent policy in a vendor-independent and device independent way. Thus, service level abstractions can be supported at a higher level, and be translated at a lower level to device-specific configuration parameters, across an aggregate of heterogeneous managed entities.

Besides the ability to structure policy rules into groups and groups of groups, PCIM introduces the notion of policy Role and component Role. The concept of role is central to the design of the entire IETF policy framework. A role is a type of property that is used to select one or more policies for a set of entities and/or components from among a much larger set of available policies [6]. Components are assigned specific roles and this helps the management system or the system administrator in selecting the appropriate policies that apply to these roles. If ever it is desired to assign a different functionality to that same element, a simple role exchange is done and the appropriate set of policies is applied accordingly. In addition, the use of roles 
enables a policy definition to be targeted to the required function of a system element, rather than to the element's type and capabilities [24].

Finally, the QoS Policy Information Model (QPIM) model builds upon PCIM (and PCIMe) a set of constructs for specifying and representing policies that administer, manage, and control access to network QoS resources [24]. It specifically deals with QoS enforcement for differentiated and integrated services using policy.

High-level business needs, available network topology, and the desired network QoS methodology such as DiffServ or IntServ drive the process of QoS policy definition in QPIM.

The following is an example illustrating the abstraction level of QPIM policies:

Example 1: Illustration of a QPIM policy abstraction level

\section{Business level policy:}

In the human resources department, applications should have better QoS than simple web applications unless it is the EXCUTIVE's web application

\section{QPIM level policy, a possible formulation:}

IF packet source or destination IP address belongs to the human resources department THEN

IF packet source or destination IP address == EXECUTIVE

THEN packet-priority $=$ High

ELSE IF packet's protocol $==$ HTTP

THEN packet-priority $=$ Low

ENDIF

ELSE packet-priority $=$ Default

On the mentioning of IETF information models, we notice when reading through PCIM(e) and QPIM a high degree of articulation in policy definition. In fact, nearly every little component of a policy is made up to be a full class. If the policy is say of the form: " $(\mathrm{Cl}$ and $\mathrm{C2}$ and $\mathrm{C3})$ or $(\mathrm{C4}$ and $C 5) \rightarrow A 1, A 2, A 3, A 4$ " then this needs to be modeled in at least ten classes! Five for the conditions, four for actions, and one for the policy itself let apart objects resulting from the instantiation of the different relationship classes. This puts the risk of having an implementation that is too slow and too much verbose. We are not really in need of this fragmentation in order to represent usual "conditions $\Rightarrow$ actions" situations. Such a fragmentation in policy representation makes it difficult for a policy administrator to modify or maintain a policy repository and makes it much harder for a policy manager to manipulate policies defined by another policy manager or even policies he had defined since long. Though this is useful at the QPIM level due to the extensive reusability nature of network level policies, this is not desirable at the human administrator specification level. A language based policy 
notation would help in improving policy readability and hence supports team based policy specification for the management of large systems.

\section{ANALYSIS AND CLASSIFICATION OF POLICY SPECIFICATION APPROACHES}

After taking a bird fly view over the "non language based" policy work under work in IETF, we will now give an account and critics of related work in the area of language based policy specification as being the focus of this article. Different approaches are considered and classified according to their abstraction level and expressiveness. The question of whether there is a "best" policy language and that of a "best" policy architecture will also be considered.

First, we will briefly review the main paradigms that have been introduced in the last fifteen years in the representation and management of network policies. The bosom of this discipline could be found in the early works on routing (such as BGP, IDPR, RPSL, and IDRP) and network management protocols. Later on, more abstract representations and models saw the light and more powerful languages were introduced ranging from natural language level specifications down to the protocol data unit level.

\subsection{Traffic flow policy specification languages}

These are relatively low-level policy languages that make use of pattern matching in the selection of the network traffic on which a policy can be applied. Their efficiency in policy-based management is limited since they do not provide a view of how the overall policy managed network system will work. A policy is simply an abstract device level representation of the functionality that a node is to hold. We mention in this category PAX-PDL [25], SRL [18], and PPL [11].

PAX-PDL and SRL codes look much like $\mathrm{C}$ code. The basic concept is the pattern, with simple patterns combined to form more complex patterns. SRL adds on pattern recognition the ability to act on the identified packet/flow by saving some statistics about it.

The Path-based Policy Language [11] was intended to bring a satisfactory solution to the integration of the IntServ and DiffServ IETF models by having a policy extended to act on the full data path of a flow. This avoids many NP-complete calculations that try to establish a coherent path based on policies spread over different nodes. Paths are analogous to static routes when they are completely instantiated. However, pre-computing paths may 
also incur excessive overhead if not designed appropriately. Yet, it is not always practical to a priori freeze paths by which a known traffic should flow.

Example 2: priority management in PPL

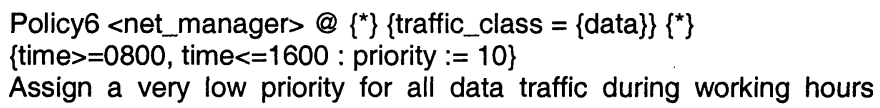

The use of userID in the grammar specification is not a needed feature and unnecessarily complicates the grammar. As also noticed by Nicodemos [2], it would better be specified as part of some meta-information that could be used for the analysis of policies.

Current traffic flow policy languages lack mechanisms for grouping policies and do not tend to take advantage of Object Oriented concepts. The search for a good policy specification language should better be done within the network wide policy specification languages, following.

\subsection{High Level Network policy languages}

They started with the elementary Clark Policy Terms [11] then many other languages followed. A policy definition is generally viewed as an "IF Condition Then Action" statement or further refined to an "On Event Then IF Condition Then Action". We will briefly describe some of those which are QoS related as we intend to leave more space for the classification part. We will also avoid some obsolete propositions like the Policy Framework Definition Language PFDL [27].

\subsection{Policy Description Language (PDL 1999)}

People in Artificial Intelligence have also been interested in policy-based management. Usually, they describe a policy as being a complete mapping from states (of the world) to actions [31]. PDL is a declarative policy definition language for system management, that is not only network systems. It formulates policies using the Event-Condition-Action (ECA) rule paradigm of active databases and extends it by providing a rich event sublanguage allowing only uninterpreted concurrent actions.

A noticeable feature of PDL concerns its rich event representation. It represents primitive (system or policy defined) events, which can be composed into complex events using boolean and temporal operators such as sequencing and alternation. An event happens in an epoch and a series of 
epochs constitute an event history. PDL has been implemented and used in some Lucent switching products.

At first glance we wondered whether adding events is a real need and that conditions are just sufficient for policy triggering. We deduced finally that this notion seems very desirable as a feature in a policy specification language. The rationale behind this concerns information that can be carried out by current status information of a system. First, as we cannot imagine that at any moment any element of a network system can be aware of the whole network state, we see events as a clever mechanism to transport useful local status information to portions of the system that need it. Second, in order to trigger a policy action we are not only in need to know about the current status of the system but we often need to know about specific evolution patterns that might have happened before and which might bring valuable information to correct policy decisions. With events we become able to mark history that we want to record and hence have a better understanding of "state" information.

\subsubsection{The Ponder Framework (1992-2002)}

Being in development since 1992 at Imperial College by Professor Morris Sloman and his research team, this policy management framework has acquired a level of abstraction and maturity that we do not find in other approaches. Compared to the other notations, Ponder has been pondered for long! And it is intended to become a universal high-level language for the uniform specification of security and management policies. Hence, we have been investigating this framework in more detail and tried to catch out the pros and cons of its actual form:

Ponder is a declarative, object-oriented language for specifying security policies with role-based access control, as well as general-purpose management policies for specifying what actions are carried out when specific events occur within the system or what resources to allocate under specific conditions. Unlike many other policy specification notations, Ponder supports typed policy specifications. Policies can be written as parameterized types, which can be instantiated multiple times with different parameters in order to create new policies. Furthermore, new policy types can be derived from existing policy types, supporting policy extension through inheritance [15]. In Ponder, special focus is put on security aspects, which are a major concern in today's increasing shift toward highly distributed systems supporting adaptive service elements.

Ponder defines policy as a rule that defines a choice in the behavior of a system [15]. This behavior is intended to reflect objectives of the system 
managers. At a more formal level, a Ponder policy defines a relationship between objects (Subjects and Targets) of a managed system.

Ponder defines four basic policy types: authorizations, obligations, refrains, and delegations and three composite policy types: roles, relationships, and management structures that are used to compose policies. Ponder also has a number of supporting abstractions that are used to define policies: domains for hierarchically grouping managed objects, events for triggering obligation policies, and constraints for controlling the enforcement of policies at runtime. All ponder constructs can be specified as single instances or as types which can be used to instantiate many instances when needed. Types in ponder can be parameterized which adds to the generality of the language.

Domains can be thought of as large file directories since they provide a means for grouping objects to which policies apply or partition objects in a large system according to some criteria. The main advantage of specifying policy scope in terms of domains is that domains may freely evolve in time with some objects being added and others removed:

Domains represent sets of objects and domain scope expressions such as those employed in the example below offer a nice flexibility to the policy action scope. This solves the problem of identifying where the policy needs to be enforced in an elegant way. A comparison of Ponder domains with IETF roles is given in 4.4 .3 on page 34 .

Obligation policies represent the key to QoS policy specification in Ponder. An obligation specifies the actions that must be performed by manager objects within the system when specific events occur and a set of conditions are met. That is, obligations are event triggered and they follow the ECA paradigm. Ponder supports a rich event composition mechanism which is much similar to that proposed in PDL. Hence, PDL ACA structures expressiveness is implicitly included into obligation policies augmented by the possibility to handle domain scope expressions for subjects and targets. Obligations support also the additional feature of exception management in the manner that we find in current Object Oriented languages. 
Example 3: application level video conference authorization [2]

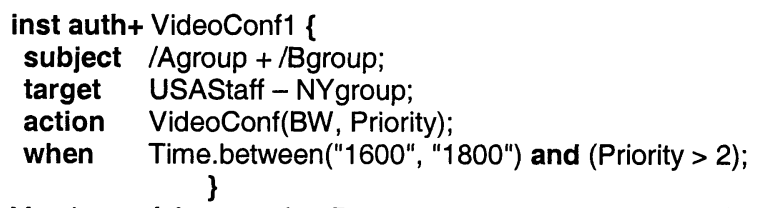

Members of Agroup plus Bgroup can set up a video conference with USA staff except the New York group. The constraint of the policy is composite. The time-based constraint limits the policy to apply between 4:00pm and 6:00pm and the action constraint specifies that the policy is valid only if the priority parameter (the 2 nd parameter of the action) is greater than 2.

Actions in ponder are object method invocations and can also be externally defined scripts stored in a certain domain. Scripts in Ponder can be implemented as objects and stored in domains where they can be accessed through authorizations.

Example 4: scripted actions in obligation policies [2]

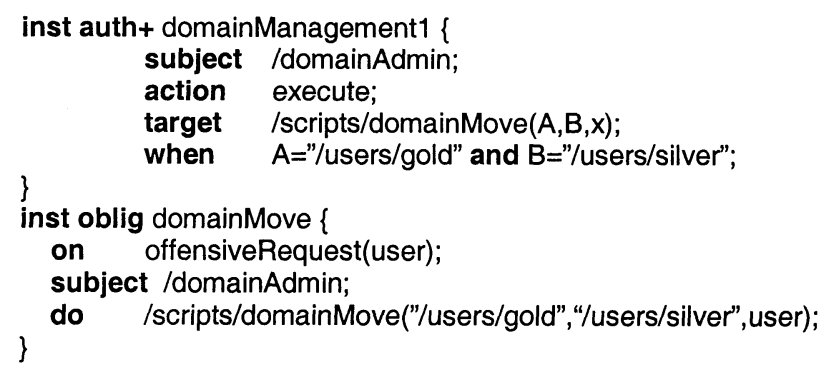

The authorization policy permits domain administrators to execute the script object 'domainMove' when A and B are "/users/gold" and "/users/silver". This script moves an object $x$ from domain $A$ to domain $B$. Because of security considerations only domain administrators are permitted to execute it, and only to downgrade gold service users to silver service users. The obligation policy specifies that domain administrators must move a user from the gold service domain ("/users/gold") to the silver service domain ("/users/silver") when that user has requested access to a web-page which is considered offensive.

Scripts in Ponder can be useful in certain cases. However, they can introduce security vulnerabilities and can cut off the uniform design of the language.

In the same vain of keeping language design uniform domains are modeled as objects in the system. A further step towards uniformity is to provide them with methods to manage objects they contain. In this way a domain could filter, using some criteria, objects it can accept. For example, a domain that is intended to reference only teachers would refuse to include a student into its referenced objects set. This feature could enhance the uniformity of the language. Hence we could reformulate the above example using this new notation: 


\section{Example 5: Enhancing Domain Representation}

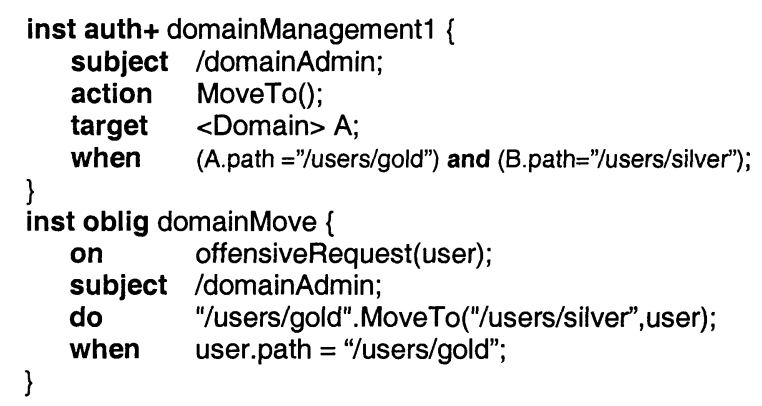

Methods concerning domain management can be defined in the Domain class. This example specifies the same functionality as that of Example 4 using a more uniform and secure manner.

Excelling other policy specification languages, Ponder offers tools for grouping policies for the sake of simplifying policy management in large complex enterprises. Four types of composite policies are provided: groups, roles, relationships, and management structures.

Roles are a major concept in Ponder. They provide a semantic grouping of policies having a common subject such as a DiffServ edge router.

Within the scope of a composite policy structure or a domain sub tree, a need exists for rules regarding permitted policy types and authorized concurrent action sequencing. For this, Meta policies have been introduced. They are expressed using Object Constraint Language (OCL) like expressions which can raise exceptions when needed. This provides a useful way to avoid policy anomalies during run time.

The ponder framework is, self managed in that policies and other constructs such as roles and relationships are implemented as objects stored within domains. Hence, they can be managed by policies stored in those domains.

Recently, there has been considerable interest in approaches used to specify higher-level policies such as those we can find in Service Level Agreements (SLA) and trust management. In its actual state, Ponder could be a candidate for enhancement in order to support SLA specifications. This will require enhancing the language with SLA structures and constructs for the definition of SLA attributes and constraints.

\subsubsection{Work on logic representation of policies}

The idea behind the turn to logic for policy representation is fundamentally linked to security and the policy conflict detection and resolution problem. Logic representations are unambiguous and more adapted to automatic consistency checking. A network policy language has 
to be flexible enough to represent a wide range of policies and at the same time formal enough to support automatic translation to logic [11]. However, Formal approaches suffer from being non intuitive and do not easily map to implementation mechanisms. They assume a strong mathematical background that makes of them difficult to use and understand. Furthermore, as we are not focused on security aspect of policy based management we will not be talking further about. Some of the languages we have described are based on formal logic grounds such as PDL while others include similar formal components like Ponder OCL expressions. However, these are surrounded by a number of non formal components which make formal analysis difficult to tackle with. The problem with formal representation of policies is that it generates NP-Complete problems which are difficult to tackle with.

\subsubsection{Policy representation in XML}

XML (eXtended Markup Language) is a rich tagged language for representing virtually any type of information. An XML document looks much like an HTML document and it has an associated Document Type Definition (DTD) which specifies the syntax that the document should conform to. Tags are always enclosed in angular braces. [1] gives an example of policy representation in XML.

Example 6: Sample XML representation of a policy

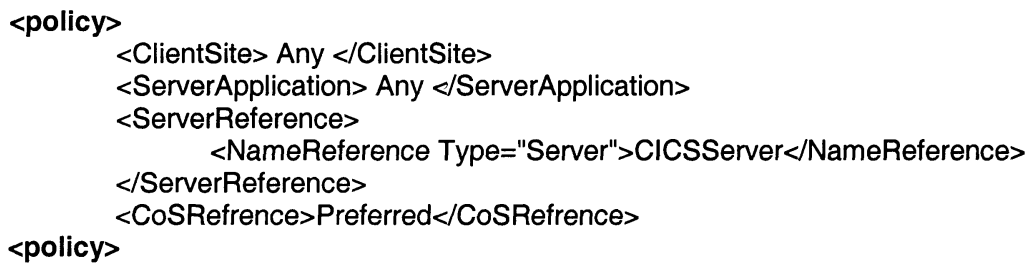

This policy specifies that any access to the transactional database system (CICS) server is a preferred communication. The DTD used to represent this policy is simple.

Figure 1: Policy representation in XML

Policy representation using XML suffers from being too verbose to be used by a policy specification agent. However, XML may be suitable for policy information exchange between the different policy management components. 


\subsection{Analysis and Classification}

We have until now reviewed a set of policy specification languages and analyzed the relative strengths and weaknesses of each. These languages broadly fall into tow categories: Abstract network policy languages such as PDL and Bit-level traffic flow languages like PAX-PDL and SRL. The main issue with low level QoS policy specification languages concerns traffic patterns manipulation. This is a desirable property which we think should be provided in high level policy specification language so that we would not require a translation to a different formalism in order to specify traffic level policies. Also, this will imply the use of the same formalism for representing both high level policies and their refined low level counterparts.

Based on the previous discussion, we identify in the following paragraphs some criteria we think are important for classifying the different policy specification formalisms we have thus far described:

\subsubsection{Supported Abstraction level(s)}

High-level policy specifications are application or business driven. They tend to be declarative. On the contrary, network level policy specification is more procedural and is nearer to the logic supported in device actions even if it specified in a device independent way.

Policy refinement cannot be automated unless the high-level abstract policy specification is done using formalisms with precise semantics. Logicbased approaches to policy specification allow formal reasoning about the specified policies, and enable properties of the specification to be proved, but they are not aimed at human interpretation and do not directly map to an implementation [2]. There have also been efforts to describe policy using natural language like expressions as is the case with the HP Power prototype [21] and the work done in [22] where is described a suite of tools that serves as an expert database management system to automate the process of mapping natural language policy statements into equivalent first-order predicate calculus. 


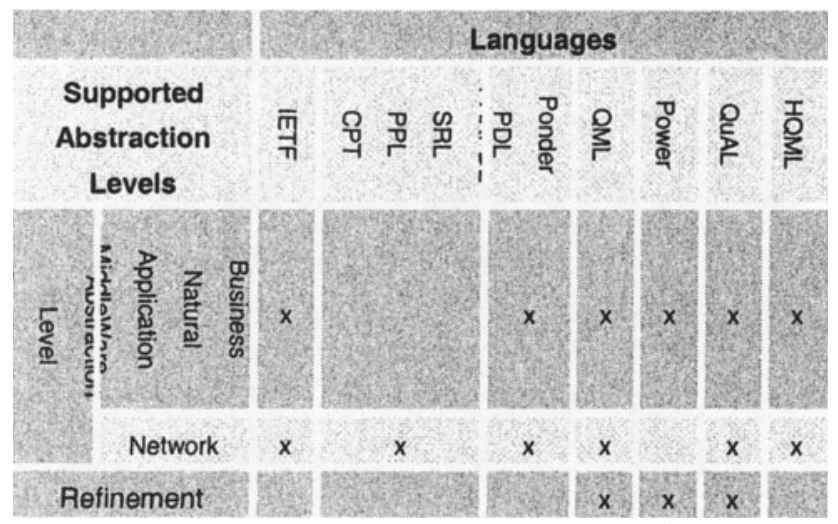

Figure 2: Supported Abstraction level(s)

The passage between an abstraction-level to another can either be TopDown, in which case it is called a refinement, or Buttom-Up which we call an abstraction. Refinement is a necessary operation that needs to be present in any High Level policy specification system. However, the abstraction of existing low-level policies into more readable high-level representations is still not addressed and the benefits of it are not clear for the moment.

\subsubsection{Formalism used to represent policies}

It is important to note that the formalism used to carry out policy notation specification plays an important role as to the expressiveness power, scalability, and usage flexibility of the policy language. Flat formalisms for example are easy to implement but are not scalable. Other tradeoffs make it difficult to support the flexibility of Object Orientation along with formal verification. 


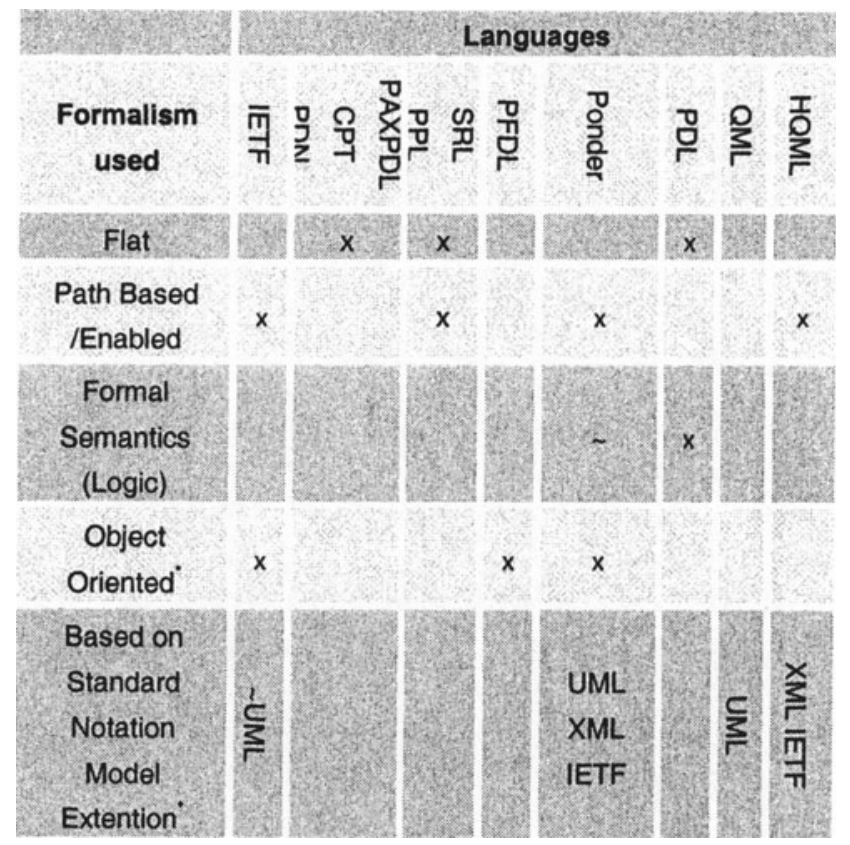

Figure 3: Formalism used to represent policy

\subsubsection{Roles, Domains, and Compatibility Rules}

The concepts of Role and Domain are very linked and are central to all the languages we have studied, though they have subtle differences. However, only two of them provide a clear definition of what a role or a domain is and how these are employed within the managed system. These are Ponder and the IETF frameworks.

- In Ponder, a Role is a semantic structure that gathers policies with a common subject, generally pertaining to a position within an organization such as a department manager. It can also specify policies that apply to an automated component acting as a subject in the system or to a network device such as a router [2]. More formally, a role is a set of authorization, obligation, refrain, and delegation policies having the same subject domain. The idea behind a role is to attach to one semantic structure all policies related to a known position in the managed system. Hence, it is possible to assign a different human or automated agent to a role without having to change the set of activities related to it since the activities are related to the role rather than to the agent assigned to it at a given time interval. 


\section{Example 7: A Ponder Role for Paris DiffServ Core Routers}

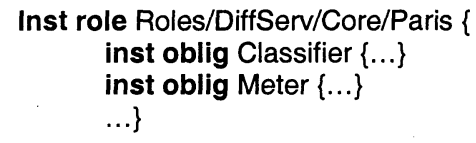

- In the IETF, a Role is a simple property. PCIM introduces the notion of policy Role and component Role. A role is a type of property that is used to select one or more policies for a set of entities and/or components from among a much larger set of available policies [6]. QPIM uses the concept of roles defined in PICM(e) to help the administrator map a given set of devices and interfaces to a given set of policy constructs. The use of roles enables a policy definition to be targeted to the network function of a network element rather than to the element's type and capabilities [24]. This helps support model scalability where a QPIM policy can be mapped to large-scale policy domains by assigning a single role to a group of network elements [24]. When collective (e.g. network) behavior must be changed, the policy administrator can perform a single update to a policy for a role, and the elements noted above will ensure that the necessary configuration updates are performed on all the resources playing that role[6].

The domain concept in IETF represents a set of network components playing the same role, where a role is simply a string attribute within an object. Role combination is specified using a " + " sign included in the Role property as is the case in Figure 4. This is very primitive compared to domain scope expressions offered in Ponder. In addition, the domain (role) concept in IETF is scattered throughout the different role strings that exist in the managed system elements and it would be difficult if not impossible to do domain maintenance operations on them.

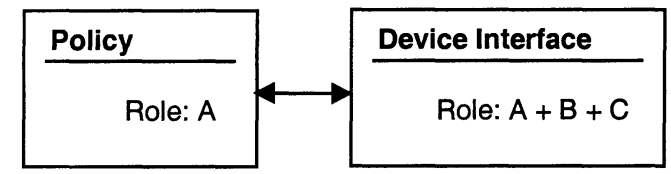

Figure 4: Roles as a key concept in policy dissemination in the IETF framework

In Ponder, Domains provide a means of hierarchically grouping objects to which policies apply and can be used to partition objects in a large system according to geographical boundaries, object type, responsibility and authority or for the convenience of human managers. A domain merely holds references to object interfaces. It can hold references to any object type, including a person. It can also reference sub-domains. Policies normally propagate to members of sub domains and domain scope expressions can be used to combine domains to form a set of objects for applying a policy. 
The main advantage of specifying policy scope in terms of domains is that domains may freely evolve in time with some objects being added and others removed. However, a design choice in Ponder was that objects have to be explicitly included in domains since it is seen not practical for performance concerns to define domain membership in terms of a predicate based on object attributes. On the other hand, a policy can select a subset of members of a domain and its sub domains, to which it applies, by means of a constraint in terms of object attributes.

Though the domain model defined in Ponder seems the best choice for our analysis, there is a feature that we think desirable to be added to them. We call this feature a Domain Membership Compatibility Rule. Building on Example 7 above, we provide these motivating samples:

Example 8: domain membership compatibility constraint

We cannot assign a DiffServ Role to a non DiffServ Enabled router. In the same way, a DiffServ Routers Domain should define specific constraints that each member must have in order to be accepted as a member of that domain.

Example 9: domain membership compatibility constraint

Router R1 can behave as a DiffServ Edge Router, DiffServ Core Router, or an IntServ Router. By stating that DSE=Routers/DiffServ/Edge is incompatible with DSC=Routers/DiffServ/Core one can ensure that R1 cannot belong simultaneously to DSE and DSC. If such rules are not defined then we could encounter this situation where R1 will receive the deployment of both a Core and Edge DiffServ router and the result of this will be unpredictable.

Example 10: abstract domain membership compatibility constraint

On Network domain D1 we have a deployed service S1. We want to deploy a new service $\mathrm{S} 2$ on domain D2. Knowing that S2 is incompatible with $\mathrm{S} 1$ (either through a property in S2 or through system manager knowledge), D2 is provided with a MembershipCompatiblity rule which forbids the inclusion of D1 objects. When D2 is created and as the needed network elements are added to it (either via a script or via the network manager) the system raises an exception if an object of D1 is being added to D2.

From the above examples, we identify a need for having domain objects or classes provided with a MembershipCompatibility predicate that each of its members must verify before being accepted members. This predicate serves as an authorization agent which controls membership to a domain. MembershipCompatibility can serve also in conflict avoidance since it disallows potential misbehavior of system elements due to their being included into a wrong domain.

Conversely, role compatibility rules can also be defined between roles (in Ponder semantics). There is a real need to know whether a device can support a given role or not as it would be as much disastrous if a device is assigned a wrong role as if it were assigned a role it cannot fulfill. Testing a device for a role to check that the device is really what it purports to be is important to the correct operation of the system. In this way, we can have a component refusing to be included into a domain because this component is not compatible with the roles that this domain supports. 
Till now, we have proposed the introduction of compatibility rules into domain and role constructs. One can also think about compatibility rules at the policy rule level. However, this provides the ability to do the same thing in many different ways, which is not always desirable in any proper uniform specification language. This issue needs to be further investigated and future work will consider the effective implementation of such compatibility rules as they will provide an additional dimension to intelligent policy conflict management.

\subsubsection{Policy Release mechanism}

We noticed that some languages base their policy triggering on special system states specified by the condition part of a IF-Condition-Then-Action policy. These languages are referred to as Proactive languages in [10]. In this case, [31] states that potential problems are detected and handled before they actually happen. This implicitly implies that in Reactive languages, i.e. event based, policies are triggered only when anomalies occur. However, this is not always the case. Events are used for more than mere anomaly reporting. Events are used to summarize particular epochs in the development of the managed system which the system manager sees as interesting. These epochs are characterized by a set of conditions that help identify them. For example, the manager may specify a policy that launches backup activities at specific time epochs:

Example 11: Daily backup policy using PDL

Event BackupTime $=$ WorkHours. End

Event BackupTime triggers SystemBackup

This same policy can be extended to take into account security considerations in presence of potential system threats:

Example 12: Augmented Daily backup policy using PDL

Event BackupTime $=$ WorkHours. End $\mid$ SystemFailureAlarm
Event BackupTime triggers SystemBackup

When events are supported in a management system, we can define more complex events based on system event history. This feature is supported by PDL and is interesting since it enables to characterize specific evolution patterns of the managed system. For example, in order to increase the security of a managed system it is useful to register previous evolution patterns that lead to system failure or some specific anomaly detection. This will help to prevent future malicious patterns to occur. 
However, basing policy triggering only on events, as is the case in Ponder for example, is not always optimal as a choice. This is illustrated in Example 13.

The problem with the policy specification in Example 13 concerns the conceptual view of a packet arrival. Since Ponder obligations only react to events, then a packet arrival cannot be represented but using an event. In the Ponder framework events are handled by a specific component called the Event Service. This means that when a packet arrives to a network node, say N1, an event say PacketArrival is generated and sent to the event service, which in its turn scans the objects concerned with the event and sends the event. Knowing that only network node N1 which is concerned with that packet, it sends the event back to it, and finally the policy actions are applied on the packet. This is just too clumsy as a conceptual view of a packet level QoS assurance mechanism. Of course, an implementation of Ponder won't do as such if has to handle network level QoS. But, this implies not following the idea of event as we know it from the language definition. Hence, we need to add a new language construct which differentiates this special type of events.

\section{Example 13: Need for Light Events}

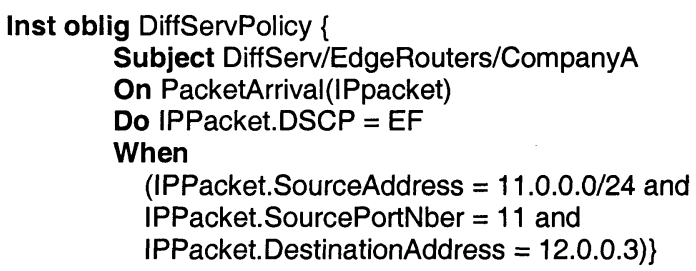

The above example tries to model Packet level QoS specification using Ponder. It also explains why using only an event based triggering mechanism for policies is not always good as a choice. Packet arrivals need to be treated locally by the network node and there is no special need to inform the event service of this.

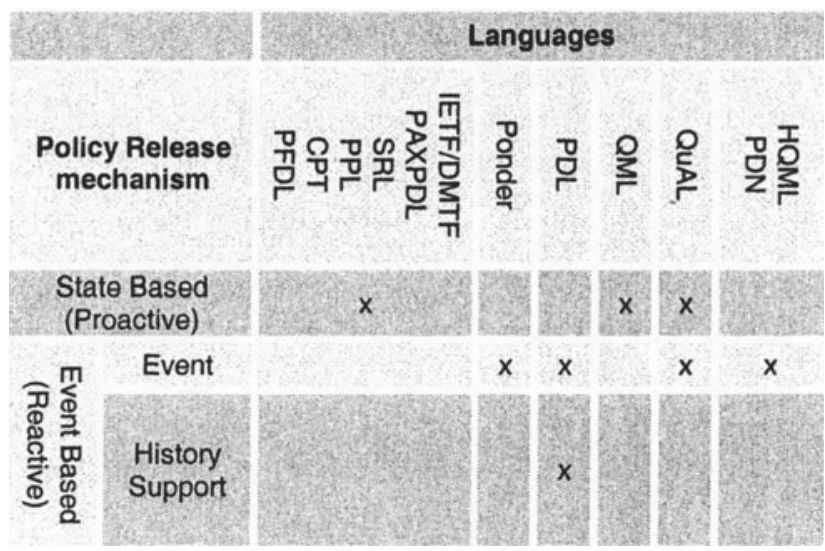

Figure 5: Policy Release mechanism

To solve this problem we propose the introduction of Light Events. A Light Event is a normal event except that it does not need to be reported to 
the event service but is rather treated locally by the relevant unit in which it occurred. Eventually, this unit may forward the event or some of its parameters or a summary of a sequence of events to the event service when this is desirable.

\subsubsection{Conflict detection/resolution}

The issue of policy conflict detection and resolution was mentioned in all language specifications as being an important component of any policy management system. If conflicting policies are deployed within a network, unexpected network behavior will occur and the damage will be difficult to expect. In fact, having a system deployed with conflicting policies is probably worse than having it without policies at all. [1] [2] [6] [24] study the issue of conflict avoidance, detection, and resolution. Conflict avoidance can be attained through the correct use of notions of domains, roles, policy compatibility, and policy structuring techniques that limit policy scope and avoid deploying a policy to the wrong system component. However, this is only stated through examples that serve as good design guides.

The definition of policy conflict depends on the approach used to specify policies. The process of policy conflict detection can only be automated when the specification is based on clear semantics. Yet, even though NPComplete problems are faced when trying to do so, as is the case with PDL. [1] shows that viewing policy rules as expert system knowledge base rules and trying to reason on them to find potential conflicts does not work. It discusses some conflict detection mechanisms in simple cases but the complexity of the operation is always deceiving and the situation is worse with real languages.

For policy conflict resolution, different strategies have been proposed. Simple Priority tags associated to the Priority attribute of the PolicyRule class have been proposed in PCIM to manage this problem. However, priority levels are generally assigned by a human administrator, which limits the automation process of policy deployment. Also, it is not evident to manage hundreds of priority tags disseminated in a distributed environment. 


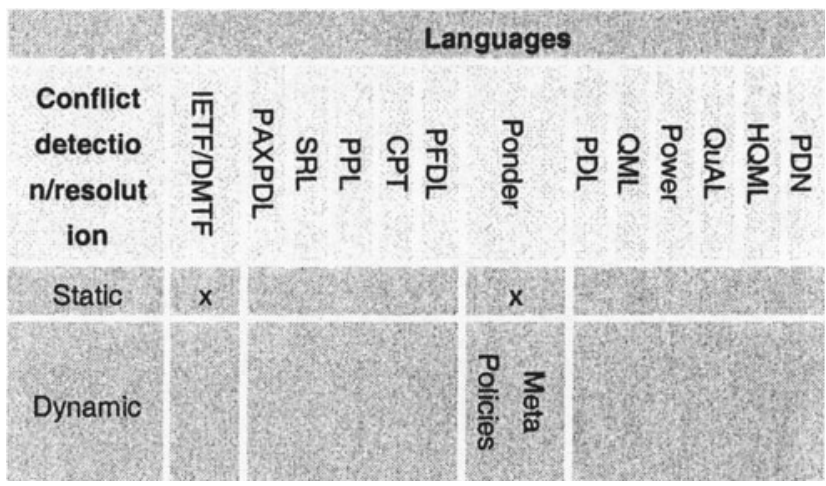

Figure 6: Conflict Detection and Resolution Facility

Nonlinear priorities are achieved via a set of metadata that define rules over the policy rules themselves, hence providing for extra control over dynamic conflict resolution. A special type of meta-data is provided in Ponder where it defines meta-policies as a structured way to define constraints within compound policy structures (Groups, Roles, Relationships, and Management Structures). Meta policies need to be defined explicitly by the policy manager as there is not an algorithm that can detect all potential conflicts that may arise at run time. In this article, we proposed an additional feature toward intelligent conflict avoidance which brings domain and role compatibility constraints.

\subsubsection{Policy life cycle and deployment model}

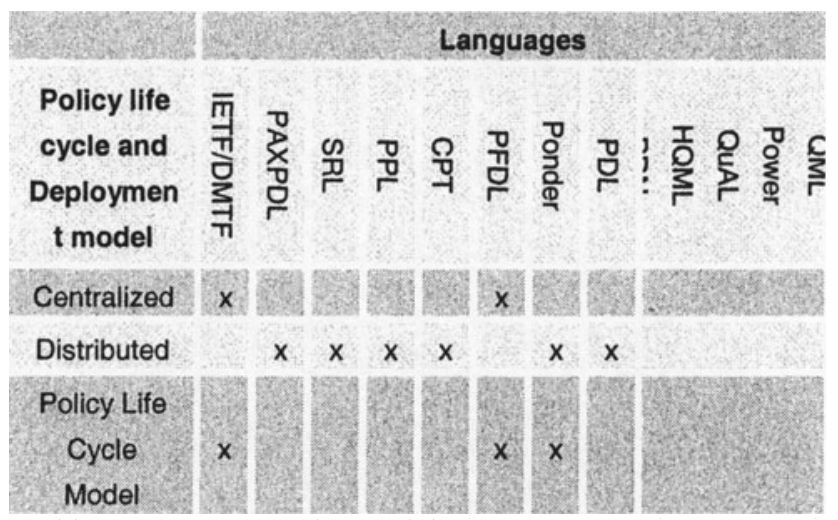

Figure 7: Policy life cycle and deployment model

In the literature, we encounter two possible deployment models for policy definition: Centralized and Distributed. The IETF defines a centralized deployment model where the PDP plays the central role in policy translation and dissemination to its PEPs. On the other hand, Ponder defines a distributed architecture for policy dissemination, which specifies more 
functional details concerning policy dissemination and policy life cycle. The distinction centralized/distributed is due mainly to the way policies are enforced. In the IETF it is the PDP that manages everything once policies are put into the policy repository. However, in Ponder, policies are enforced using autonomous agents that can interact with both the event and domain services at run time.

\subsubsection{Concluding remarks and future work}

A Network QoS policy specification language is designed to help a network administrator enter easily the needed QoS decisions or rules of behavior that are desired for the proper functioning of the network. Hence, this language should be easy enough to be used by a human administrator and at the same time strong enough to be able to specify any kind of QoS policy requirement as well as possibilities for future extensions of those requirements. Through our study, we noticed that the domain of policy based management has gained in maturity in the last decade where it moved from simple low packet level filtering specifications centered over individual components, then there came higher level policy specification languages offering more structures and targeting the management of the whole system, then there has been attempts to specify policy using natural languages, and today we are witnessing the emergence of commercial tools offering policy based management solutions to real scale management systems. However, there is still much to do in policy-based management and in policy notation. The existing commercial solutions are accused of being inefficient although they offer convivial graphical interfaces and ease of use. Those solutions are generally based on IETF policy schemas but are characterized of being single-vendor approaches and are also accused of being less efficient than expected as they do not employ all the desired features we employed. As such, they cannot be easily employed in existing multi vendor networks. Succeeding to define a high-level vendor independent policy specification framework should be the right approach for simplifying and unifying the management of complex networked systems.

In our study we could identify a set of useful constructs that are desirable to be present in a practical high-level policy specification language and proposed some new features, which we hereby summarize:

- Event-Condition-Action (ECA) rules for event triggered management actions and provision of Light Events in cases where full event mechanisms may slow down system performance (page 34). The support of Event Histories as in PDL helps in identifying specific evolution patterns that might have happened before and which might bring valuable information to correct policy decisions. Ponder and PDL offer ECA rules in a more natural way (as predicates). However they need to be adapted to support packet level pattern specifications, such as in PAX-DPL, in order to allow specification of network level QoS policies. 
- Structuring techniques to promote policy specification scalability in large systems. This requires the ability to apply policies to large collections of objects. In this context, the work undertaken in IETF for the representation of the different managed entities is very useful. Besides, the use of domains to group those entities facilitates the specification of subject and target entities addressed by policy. Specifying policy scope in terms of subjects and targets is useful in identifying the context of policy and helps in policy analysis.

- Policy Composition structures to group together policies relating to some criteria. Composite policies are essential to cater for the complexity of policy administration in large enterprise information systems. The IETF provides the PolicySet class for policy grouping and does also allow the composition of conditions and actions into complex hierarchies. Ponder provides a more specified way for policy grouping in terms of Groups, Roles, Relationships, and Management Structures. The two structuring techniques are not incompatible and could be combined into a same specification language.

- Reusability of existing definitions. This is accomplished via the use of object-oriented concepts in policy definition. Both IETF and Ponder use Object Oriented concepts. Ponder offers also policy class definitions which it names as policy types and it does not allow specialization of policy classes while it allows specialization of composite policy structures. Thinking of a policy as a class is useful for deriving more specific policies and reusing existing design. Allowing policy definitions to contain attributes and methods is also important. For example, complex actions can be specified separately in a method and then called in the action part of the policy. Attributes can also serve to characterize specific values to be used in the condition part.

- Conflict detection/resolution in the specification and in run time. Declarative language design, object structuring techniques, and the use of compatibility rules participate in reducing the potential of conflicting design as performance trade-offs (4.4.5) limit the use of formal logic in solving this problem.

- Extensibility to cater for new policy types that may arise in the future. This is implicitly offered by the object-oriented design.

- Ease of use of the language. It must be comprehensible and easy to use by policy users. For all languages we have studied, the policy manager needs special skill in order to specify policy. Ease of use can be provided by developing graphical interfaces for policy definition and/or building a natural language processing layer which translates naturally specified expressions into structured policy terms. Work on POWER [21] serves in this vain. However, more important is the notation we use for technical 
policy specification before going up to natural language policy specification.

- An additional interesting feature concerns self-management introduced in Ponder [2] where policies are used to manage not only other system components but also system policies them selves. This allows the uniform automation of activation, deactivation, and redefinition of policy objects via policy objects.

In the scope of our research, we identified that there are two major policy specification frameworks which merit special attention, i.e. Ponder and the IETF, and we discussed the pros and cons of each along with other existing frameworks. The Ponder framework has not yet been tested in a real-scale system, as is the case with PDL, since a number of its features have been found difficult to implement. However, this does not lure out the fact that it is nicely designed and, moreover, is available as open source. Based on this, future work will consider the combination of those two approaches and testing of their applicability in managing different QoS specification levels starting by SLA/SLS contracts specification and ending up with the specification of network level policies for a QoS enabled network (DiffServ, IntServ, or MPLS). In such an endeavor, we will further develop and implement the notions of domain membership compatibility rules, role compatibility rules, and light event structures we have motivated in this research effort.

\section{REFERENCES}

[1] Dinesh C. Verma, New Riders, Policy Based Networking, Architecture and Algorithms, New Riders, USA, Nov 2000

[2] Nicodemos Damianou, A Policy Framework for Management of

Distributed Systems, PhD Thesis, Imperial College of Science, April 2002.

[3] I. Aib, M.S. Fonseca, G. Pujolle, B. Campedel, H. Chaouchi, Wissam

Fawaz, Analysis of Models and Specification Languages for Policy Based

Management of Networks and Distributed Systems, DEA Thesis, LIP6 Laboratory, University Paris 6.

[4] A. Westerinen, J. Schnizlein, Cisco Systems, J. Strassner, Intelliden Corporation, et al, "Terminology for Policy-Based Management", IETF, RFC 3198, November 2001, http://www.ietf.org/rfc/rfc3198.txt?number=3198.

[5] B. Moore, IBM, E. Ellesson, LongBoard, Inc, J. Strassner, A.Westerinen, Cisco Systems, "Policy Core Information Model, Version 1 Specification", IETF, RFC 
3090, February 2001, available from

http://www.ietf.org/rfc/rfc3060.txt?number=3060.

[6] B. Moore, Editor, "Policy Core Information Model Extensions.

(PCIM)", IETF, RFC 3060, IETF, draft-ietf-policy-pcim-ext-08.txt, May 2002.

[7] DMTF DSP0108, "CIM Core Policy Model”, DMTF, May 122000.

[8] DMTF, “CIM Core Model White Paper, Version 2.4", DMTF, 2000, www.dmtf.org.

[9] IETF Network Working Group, "Key words for use in RFCs to indicate Requirement Levels", IETF, RFC 2119, 1997.

[10] Gladys Diaz, Sandrine Duflos, Valérie Gay, Eric Horlait, "A comparative study of Policy Specification Languages for Distributed Applications", Submitted for publication to the $13^{\text {th }}$ IFIP/IEEE International Workshop on Distributed Systems: Operations and Management (DSOM 2002), August 2002.

[11] Gary N. Stone, Bert Lundy, and Geoffrey G. Xie, U.S Department of Deffense, "Network Policy Languages: A Survey and a New Approach", IEEE Network, January/February 2001.

[12] Jorge Lobo, Randeep Batia, Shamim Naqvi, (Bell Labs), “A Policy Description Language",

American Association for Artificial Intelligence (www.aaai.org), 1999.

[13] Leonidas Lymberopoulos, E. Lupu, M. Sloman, "An Adaptive Policy Based Framework for Network Services Management", Imperial College, Proc. $3^{\text {rd }}$ IEEE Workshop on Policies for Distributed Systems and Networks (Policy 2002), Monrerey, California, June 2002, pp147-158.

[14] Morris Sloman,Wayne Luk,Emil Lupu, Naranker Dulay, "Polyander: Language Based Policy Specification, Analysis and Deployment for Large-scale Systems". Cisco University Research Programme, September 2001.

[15] N. Dulay, E. Lupu, M. Sloman, N. Damianou, “A Policy Deployment Model for the Ponder Language", An extended version of paper in Proc. IEEE/IFIP International Symposium on Integrated Network Management (IM'2001), Seattle, May 2001, IEEE Press.

[16] Nicodemos Damianou, Naranker Dulay, Emil Lupu, Morris Sloman, "The Ponder Policy Specification Language", Department of Computing, Imperial College, V5 2/8/2000.

[17] Svend Frolund, Jari Koistinen, "Quality of Service Specification in Distributed Object Systems Design", HP Laboratories.

[18] Nevil Brownlee, IETF August 1999, "SRL: A Language for Describing Traffic Flows and Specifying Actions for Flow Groups", IETF, http://www.watersprings.org/pub/id/draft-ietf-rtfm-ruleset-language-07.txt. [19] "CIM Tutorial”, DMTF, http://www.dmtf.org/education/cimtutorial.php, March 2, 1999. 
[20] Hugh Mathon, Yoram Bernet, Shai Herzog, Jhon Schnizlein,

"Requirements for a policy management system", IETF work in progress, draft-ietf-policy-req-02.txt.

[21] M. Casassa Mont, A. Baldwin and C. Goh, "POWER Prototype:

Towards Integrated Policy-Based Management", HP Labs Technical Reports HPL-1999-126, 18/10/1999.

[22] Michael, J. B., V. L. Ong and N. C. Rowe (2001). "Natural-Language Processing Support for Developing Policy-Governed Software Systems". In Proceedings of the $39^{\text {th }}$ International Conference on Technology for Object-Oriented Languages and Systems, Santa Barbara, California, IEEE Computer Society Press, July 2001, pp. 263- 274.

[23] Oscar Diaz Alcantara, Morris Sloman, "QoS policy specification - A mapping from Ponder to the IETF", Department of Compouting, Imperial College, 180 Queen's Gate, London SW7 2BZ.

[24] Y. Snir, Y. Ramberg Cisco Systems, J. Strassner Intelliden, R. Cohen Ntear LLC, B. Moore IBM.

"Policy QoS Inofrmation Model" (QPIM),

IETF Internet Draft, Expired May 2002.

[25] Solidum, "PAX Pattern Description Language Reference Guide", http://www.solidum.com/products/pax_pdl.cfm, April 2002.

[26] John Strassner, "Mapping the Policy Core Information Model to a Directory", DEN SEVT, Portugal, December 2000.

[27] John Strassner, Stephen chleimer, "Policy Framework Definition Language",

IETF draft-ieft-policy-framework-pfdl-00.txt, 1998.

[28] Zhao W, Olshefski D, Schulzrinne H, "Internet Quality of Service: an Overview", Technical Report CUCS-003-00. http://www.cs.columbia.edu/ hgs/netbib/, (2000).

[29] C. Baral, M. Gelfond, A. Provetti. "Representing Actions: Laws, Observations and Hypothesis". Journal of logic Programming 31 (1-3), 1997, pp. 201-244.

[30] C. Baral, J. Lobo, G. Trajcevski, "Formal characterizations of active databases: II". In Proc. Of the international conference on DOOD, 1997.

[31] H. Lufiyya and al., "Managing QoS requirements", UWO Technical report 547, November, 1999.

[32] E. C.Lupu, A Role-Based Framework for Distributed Systems Management, $\mathrm{PhD}$ Thesis, Department of Computing, Imperial College. London, U.K., July 1998.

[33] Morris Sloman. "Policy Driven Management for Distributed Systems", Journal of Network and Systems Management, vol. 2(4), pp. 333-360, December 1994.

[34] S. Russel, P. Norvig, Artificial Intelligence-A modern approach, Prentice Hall, 1995. 